\title{
TheChildLab.com
}

\section{A Video Chat Platform for Developmental Research}

\author{
Mark Sheskin $^{1 *} \&$ Frank C. Keil ${ }^{2}$ \\ ${ }^{1}$ Cognitive Science Program, Yale University, New Haven, CT, USA \\ ${ }^{2}$ Psychology Department, Yale University, New Haven, CT, USA
}

*Corresponding Author:

Mark Sheskin

2 Hillhouse Avenue

Yale University

msheskin@gmail.com 


\begin{abstract}
Over the past decade, the internet has become an important platform for many types of psychology research, especially research with adult participants on Amazon's Mechanical Turk. More recently, developmental researchers have begun to explore how online studies might be conducted with infants and children. Here, we introduce a new platform for online developmental research that includes live interaction with a researcher, and use it to replicate classic results in the literature. We end by discussing future research, including the potential for large-scale crosscultural and longitudinal research.
\end{abstract}




\section{TheChildLab.com}

\section{A Video Chat Platform for Developmental Research}

\section{Introduction}

Over the past decade, the internet has become an increasingly important part of psychology research, both as (1) a way for large numbers of people to participate in experiments, and as (2) a way for researchers around the globe to collaborate. Regarding inclusion of large numbers of participants, the most notable example is Amazon's Mechanical Turk platform, which matches tens of thousands of adult participants with studies from researchers around the world (Bohannon, 2016). More recently, developmental researchers at MIT have begun pilot testing a platform, "Lookit," that allows parents and children to participate in developmental studies. In a paper describing the Lookit platform, the authors identify four key advantages to conducting developmental research online: increasing sample size, reaching special populations, scheduling repeated sessions, and supporting replication by facilitating the standardization and sharing of protocols (Scott \& Schulz, 2017). In an initial set of studies establishing the utility of computeradministered tasks online, Lookit was used to collect looking time data with infants and verbal response data with three- and four-year-olds (Scott, Chu, \& Schulz, 2017).

Regarding collaboration between researchers, multi-site collaboration has become increasingly common across many scientific disciplines in recent decades (Wagner et al., 2017), and several groups have proposed multi-site collaborations in which many research groups collaborate by each collecting a (relatively) small amount of data. For example, multiple papers have been published as part of a "Many Labs" collaboration (e.g., Ebersole et al., 2016; Klein et al., 2014). A recently-announced initiative called "ManyBabies" aims to apply the same ideas 
behind "Many Labs" to infancy research, which is especially valuable due to the difficulty of any individual infant lab collecting large datasets. An initial paper (Frank et al., 2017) has described goals and initial protocols for ManyBabies, with an initial proof-of-concept replication study (on infant preferences for infant directed speech) in progress.

The emerging technologies for participants and researchers to connect over the internet in large numbers have emerged at a critical time during discussions of best practices in psychological science. For example, both the increase in the number of available participants and the ability to share the exact online presentation of a study are in line with an increasing focus on large samples and replication (Open Science Collaboration, 2015; Simmons et al., 2011). As another example, the ability to recruit participants from diverse backgrounds around the world is in line with the increasing focus on the importance of investigating when and how there is cross-cultural variation in results (Henrich et al., 2010), rather than relying almost exclusively on research with participants from a small number of Western Educated Industrialized Rich Democratic (that is, "WEIRD") countries.

\section{TheChildLab.com}

In contrast with computer-administered studies that do not involve live interaction with a researcher (e.g., Lookit), our goals were to develop a video chat platform to be used for researcheradministered studies, and then to provide initial validation of the platform by using it to replicate standard results in the developmental literature. Although studies administered by a researcher are more time-intensive than studies that can be administered by a computer (as at Mechanical Turk for adults and Lookit for children), some studies that are not well-suited for computeradministration can be administered over video chat. 
For such studies, there are many potential advantages to recruiting and running participants over the internet. Some of these overlap with advantages of computer-administered online tasks: reaching specific populations, scheduling repeated sessions, and facilitating replication by facilitating the standardization and sharing of protocols. At least one advantage-increasing sample size — is present but decreased compared to an automated platform (i.e., in contrast with an on-demand study administered by computer, in a live video chat the total number of children that can participate with a particular researcher each day is limited by that researcher's availability).

On the other hand, a live interaction with a researcher allows for study designs not otherwise possible, including allowing the researcher to conduct the study in a consistent way rather than relying on each parent to do so. In addition, compared to an in-person interaction, conducting a live interaction via video chat allows researchers to remain blind to condition manipulations by showing pictures or videos only to the participating child, and not to the researcher.

\section{The Current Studies}

To validate that our video chat platform is suitable for running standard types of developmental studies, we attempted to replicate effects that children over 5 years old generally show: false-belief understanding in the Sally-Anne task (Baron-Cohen, Leslie, \& Frith, 1985); equal division of resources between third parties (e.g., Sheskin et al., 2016), and following the path of a falling object through a tube rather than straight down (e.g., Hood, 1995). As a training item, we also included a pair of simple memory questions. Across all four of these tasks, there were a total of six questions of interest (one Sally-Anne, one Fairness, two Gravity Tubes, two Memory). 


\section{Method}

The method for this study, including the PowerPoint file for one of the counterbalances, was pre-registered at the Open Science Foundation website, at this link: osf.io/xdnuw.

\section{Participants}

Participants were recruited via a database of parents who had signed up at TheChildLab.com to be informed when we had a study available for their child's age group. The e-mail inviting a parent to participate in the study included this information about compensation: "Children who participate in person get to choose a prize; since your child would be participating online, we would e-mail you a \$10 gift card to Amazon.com.” To participate, families needed to have an internet- and webcam-connected device, which included a wide range of desktops, laptops, tablets, and smartphones.

We pre-planned a sample size of 50 children, including at least 10 children in each of four two-year age spans (5- and 6-year-olds, 7- and 8-year-olds, 9- and 10-year-olds, 11- and 12-yearolds). Our recruitment yielded fewer children in the older age groups than we expected: when we reached our target of 50 children, the numbers in each two-year span were, starting with the youngest (5- and 6-year-olds): 16, 22, 7, and 5. Since we were most interested in validating that our platform worked with children at the younger end of our age range, we did not pursue supplemental data collection to fill out the oldest age brackets. We did not have any exclusion criteria, and all 50 children were included in all analyses.

\section{Procedure}


The researcher began each session with an informed consent process approved by our Institutional Review Board. In describing the study to the parent and child, the child was introduced to the standardized format we used (with some variation) for each task (see Figure 1). Our intention with the standardized format (e.g., color-coded answers choices) was to ease the cognitive load of the tasks and to provide clear expectations for when the child was expected to answer (which we expected might be particularly important when there was connection lag).

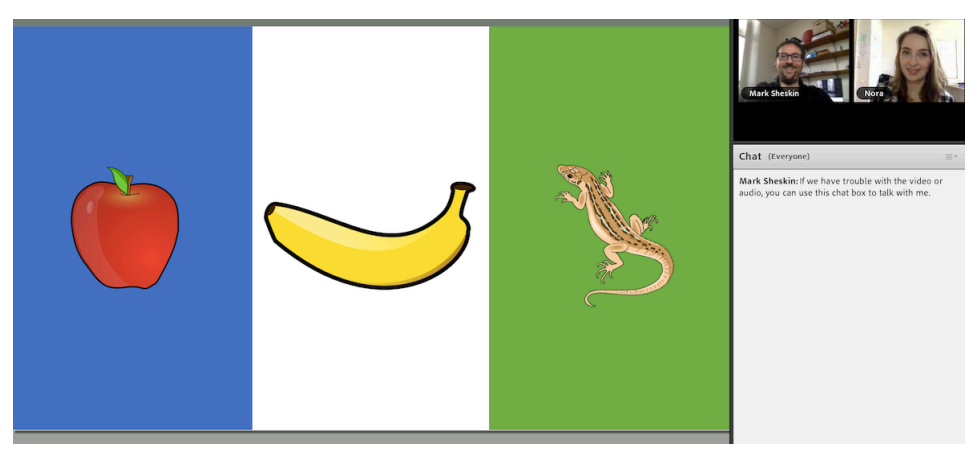

Figure 1. The Video Chat Interface. The screen can be flexibly arranged between chat windows (here shown with two researchers, small in top right), text chat (bottom right), and shared picture or video content (left). Most of the questions were asked while the main part of the screen was split between three bars, with the questions illustrated in the central white bar and potential answers (color coded "blue" and "green") shown on either side of it.

After the researcher described the studies, answered any questions from the parent or child, and confirmed that the child assented and the parent intended to consent, the parent was then asked to audio record consent by reading through a script displayed onscreen. To keep names and answers separate, the study session itself was recorded in a separate audio file. To ensure data accuracy, a second researcher coded the data from the audio file.

After consent, training questions began by asking children to say "blue" or "green" as each bar appeared in isolation several times (in the pattern blue, green, blue, blue, green, green). This 
ensured that each child knew how to respond to the questions, and emphasized that each answer would typically be one of these responses. Two memory questions (see below) were used as additional training, and the other tasks were presented in two counterbalanced order, either "SallyAnne, Fairness, Gravity Tubes" or the reverse. For each of the two task orders, the correct answer was counterbalanced for each question, appearing in "blue" for half of the children and "green"

for half of the children. The combination of task counterbalancing and answer counterbalancing produced a total of eight versions such that the researcher-who asked the questions but did not see the answer choices being displayed to a particular child-could not predict "correct" answers based on previous answers (e.g., a child answering "blue" for the first question provided no information about the "correct" answer on the next question).

\section{Memory Questions}

The first questions for each child were a pair of memory questions (see Figure 2). In one question, children saw a small blue circle and a larger green circle (the radius of the green circle was twice that of the blue circle, though the exact sizes were dependent on screen size), and needed to remember which circle was larger after the circles disappeared. In the other question, children saw a $4 \times 2$ grid containing ten blue circles and two green circles and needed to remember, after the circles had disappeared, whether there had been more blue or more green circles.

These questions also served as additional training to emphasize that information would need to be remembered, rather than always being visible on screen when a question was asked. To achieve this goal, the experimenter was not blind to the correct answer and re-showed the circles if the child did not answer correctly the first time. A child was coded as getting a memory question 
correct if the correct answer was given on the first try, without the experimenter reshowing the circles.

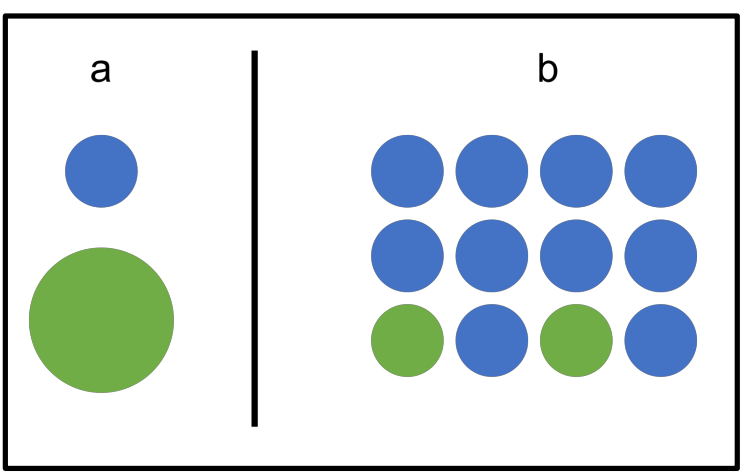

Figure 2. The Two Memory Questions. Children always saw (a) first, and were asked to note how big each circle was, but the circles disappeared before the experimenter asked if the blue circle or green circle was bigger. Children always saw (b) next and were asked to note how many blue circles and green circles there were, but the circles disappeared before the experimenter asked if there were more blue or green circles.

\section{Sally-Anne False Belief Task}

The Sally-Anne false belief task was adapted from the original black-and-white stimuli (Baron-Cohen et al., 1985). So that the researcher could refer unambiguously to each character, the characters' shirts were made different colors and were introduced to each child with this information highlighted "Here are two girls. Sally is in the yellow shirt, and Anne is in the red shirt." So that the researcher could refer unambiguously to each location, and to allow the researcher to remain blind to which location Sally put her ball (counterbalanced across children), both locations were colored boxes (see Figure 3 ). 


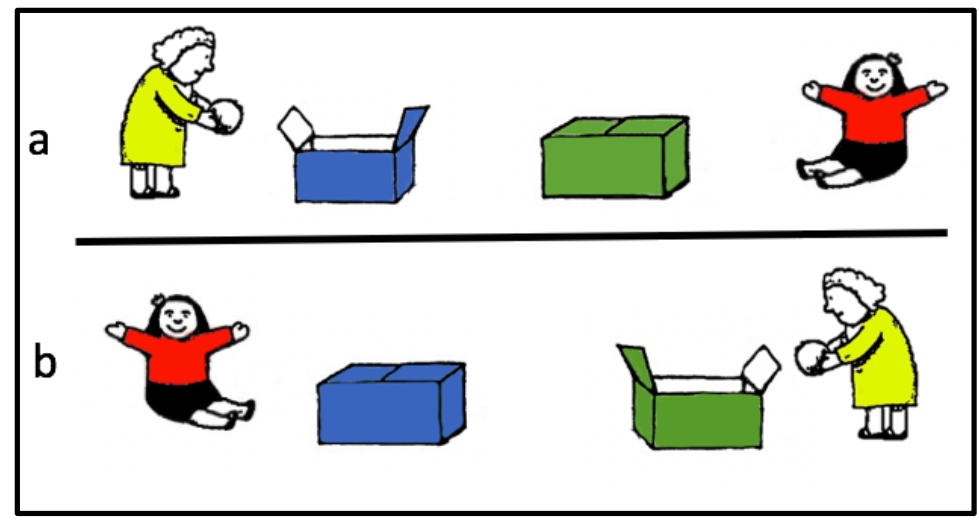

Figure 3. The Sally-Anne Task. In this critical scene from the story, Sally hides her ball in either the blue box (a) or the green box (b). The researcher does not know which version any particular child sees, and for either one says "Sally has a ball, and she puts the ball in one of the boxes."

\section{Fairness Question}

The fairness question asked children to decide how the researcher should give two stickers to two recipients: one for each recipient, or both for one recipient and zero for the other recipient. As with the Sally-Anne task, the side of each answer was counterbalanced across participants and the researcher remained blind to condition, saying "Look at how the stickers are in blue, and look at how the stickers are in green. How should I give the stickers to the girls? Blue, or green?" (see Figure 4).

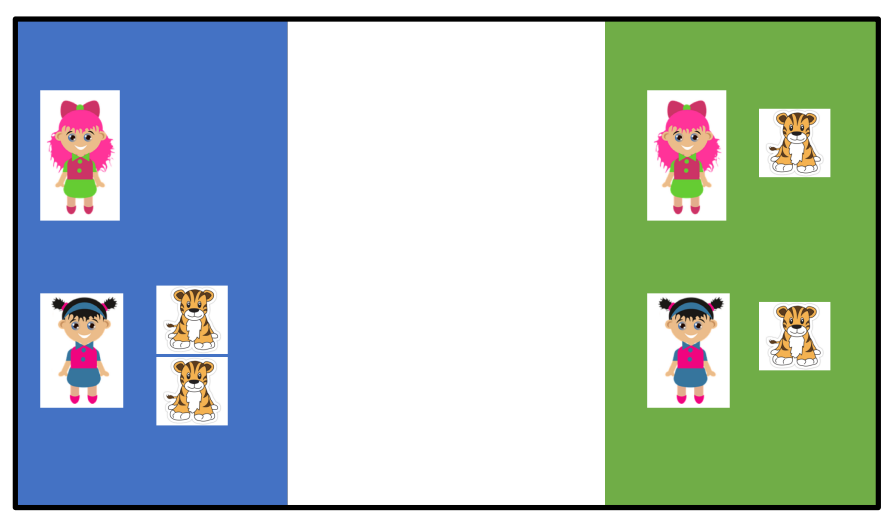


Figure 4. The Fairness Question. Children chose between an unfair distribution of "zero stickers for one person and two stickers for one person" and a fair distribution of "one sticker per person." The options were not verbally labeled, because the researcher was blind to which option was on which side for each child.

\section{Gravity Tube Questions}

The gravity tube questions were adapted from pictures of the physical stimuli used in the original study (Hood, 1995). As with the other questions, the experimenter remained blind to counterbalance and asked "Where do you think the red ball will go? Blue, yellow, or green?" These questions also included two features not present in the previous ones. First, they included animations (the ball falling into the tube). Second, the answer format included three (rather than two) choices (see Figure 5).

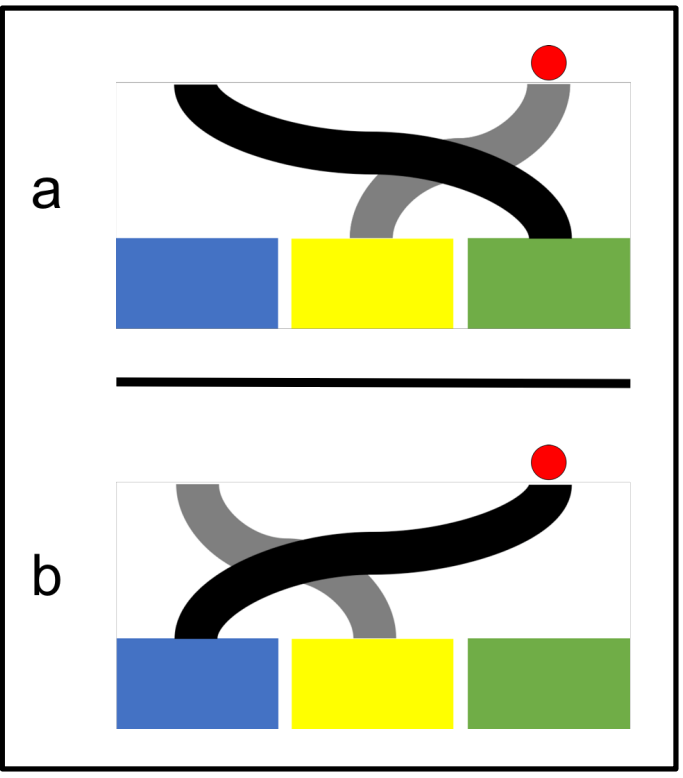


Figure 5. The Two Gravity Tasks. Children saw (a) and (b) in a counterbalanced order, so that the researcher did not know (especially for the first one a child saw) whether the expected answer was "blue" or "yellow."

\section{Results}

The raw data are available at the same link as the pre-registration: osf.io/xdnuw Children provided the expected answer on all six of the questions (binomial tests, $p \mathrm{~s}<.05$ ). For five of the six questions, performance was particularly strong $(p \mathrm{~s}<.001)$ : out of 50 children, the expected answer was given by 48 in the Size Memory question, 49 in the Number Memory question, 46 in the Fairness question, 47 in Gravity Tube A, and 48 in Gravity Tube B. In contrast, performance on the Sally-Anne task was significant, but weaker, with 33 of 50 children giving the expected answer $(p=.016)$.

\section{Post-hoc Analyses}

We did not perform any post-hoc analyses on the five questions for which nearly every child answered as expected, since this pattern of results means there is almost no variation to investigate. In contrast, we performed a post-hoc analysis to investigate two potential causes of variance on the Sally-Anne question: child's age, and whether the child received a counterbalance in which the question appeared relatively early (order: Memory Questions, Sally-Anne, Fairness, Gravity Tubes) or relatively late (order: Memory Questions, Gravity Tubes, Fairness, Sally-Anne). We thought it was plausible that order might influence performance in either direction: children who received a challenging question later might do better because they had more practice with the online study format, or might do less well because of fatigue and distraction. 
A binary logistic regression revealed a main effect for age $(p=.038)$. There was no effect for order $(p=.809)$, and there was no interaction effect $(p=.900)$. Looking at performance by two-year age groupings, the "false belief" location was given by $44 \%$ of 5 - and 6-year-olds (7 of 16 children, binomial, $p=.773$ ), 64\% of 7- and 8-year-olds (14 of 22 children, binomial, $p=.143$ ), $100 \%$ of 9 - and 10 -year-olds ( 7 of 7 children, $p=.008$ ), and $100 \%$ of 11 - and 12 -year-olds ( 5 of 5 children, $p=.031)$.

\section{Discussion}

Children participating in developmental research via a video chat platform provided expected answers on a variety of tasks, from social tasks presented with static images (e.g., our fairness question) to causal reasoning tasks presented with animations (e.g., our gravity tube questions). We also identified at least one task that might be harder (our implementation of the Sally-Anne Task). Future research can establish best practices for asking questions via video chat, including which features make them more or less difficult. For example, it could be that presenting the Sally-Anne story images one at a time, synched to the narration from the researcher, was difficult to follow for some children, especially if the timing between audio and video moved out of synch. In general, there might be tasks that are inherently more difficult in an online format compared to in a traditional face-to-face interaction, but there may also be tasks that are easier in an online format. For example, in tasks that might naturally be run on a laptop (e.g., in a museum, with many distractions nearby including the physical presence of a researcher), an online format may allow children to complete the tasks in a familiar environment with fewer distractions.

Future research should also investigate diverse approaches to study format. Although we have emphasized the advantages of a standard question and response format, a video chat platform 
is not limited to the exemplars we report here. Indeed, beyond these pre-registered studies, we have also piloted a wide range of question and response formats, including streaming pre-recorded video (of a puppet show) and asking open-ended questions. Additional research will establish which formats have sufficient reliability to be a good match for video chat.

One possibility is that there will be some formats that work better for some participants than others. For example, streaming a video that includes important small-resolution details requires a high-bandwidth connection but does not require a low-lag connection, whereas a backand-forth free-form conversation can take place over lower bandwidth but requires very low lag. When studies have specific requirements for connection quality, researchers can assess important features like bandwidth and lag at the beginning of a session (or have this information available from a previous session) and then run a study that is the best match for the participating family.

Other research might investigate the role of parental proximity. Parents might inadvertently influence children's responses merely by being present (e.g., observer effects, Engelmann, 2013; though see Warneken \& Tomasello, 2013). In the current studies, parents typically sat beside the child. However, the potential for parental influence can be eliminated by asking the parent to not be visible to the child during the study. Although this cannot be achieved merely by having the parent sit behind the child (the parent would still be visible in the video chat window), it can be easily achieved when families have two internet-connected devices: the child can then participate using one device (e.g., laptop) while the parent observes the interaction — but is not visible in itwith another device (e.g., smartphone) in another room.

Future research with our platform can also include a focus on collaboration across large physical distances. As discussed in the paper introducing ManyBabies (Frank et al., 2017), "Collaborative efforts provide significant opportunities for networking, mentorship, and the 
sharing and crossfertilization of ideas, well beyond those afforded by the standard conference and publication paradigm. With the widespread use of videoconferencing, collaborative projects bring together researchers across timezones in relatively intimate, friendly, supportive, and significant interactions."

Finally, an online platform can be used for including additional participants from both WEIRD and non-WEIRD populations (Henrich et al., 2010). For participants who live far away from most developmental researchers, an online video chat platform allows researchers to stay in one location and nevertheless include participants from around the world (rather than needing to be present locally, e.g., House et al., 2013; Safra et al., 2014). Although our current infrastructure can only include parents who have access to internet-connected devices at home, future research might also include families without home internet access. For example, researchers could partner with a local school in a developing area and pay to install a computer and internet access, with a volunteer or employee at the school coordinating scheduling and compensation of interested families. Importantly, the online environment can facilitate greater standardization across samples even when there is wide variation in geographic location or which researchers are involved.

In sum, we have found that children provide expected responses on several classic tasks asked via a video chat platform. Further research will reveal additional benefits and limitations of this platform, as well as best practices. As with data collection with adults, an online platform will not be the best option for all studies—-but we are excited to continue to learn the types of studies for which it can be a useful approach. 


\section{References}

Baron-Cohen, S., Leslie, A. M., \& Frith, U. (1985). Does the autistic child have a "theory of mind"?. Cognition, 21(1), 37-46. https://doi.org/10.1016/0010-0277(85)90022-8

Bohannon, J. (2016). Mechanical Turk upends social sciences. Science, 352(6291), 1263-1264. https://doi.org/10.1126/science.352.6291.1263

Ebersole, C. R., Atherton, O. E., Belanger, A. L., Skulborstad, H. M., Allen, J. M., Banks, J. B., ... \& Brown, E. R. (2016). Many Labs 3: Evaluating participant pool quality across the academic semester via replication. Journal of Experimental Social Psychology, 67, 68-82. https://doi.org/10.1016/j.jesp.2015.10.012

Engelmann, J. M., Over, H., Herrmann, E., \& Tomasello, M. (2013). Young children care more about their reputation with ingroup members and potential reciprocators. Developmental Science, 16(6), 952-958. https://doi.org/10.1111/desc.12086

Frank, M. C., Bergelson, E., Bergmann, C., Cristia, A., Floccia, C., Gervain, J., ... \& LewWilliams, C. (2017). A Collaborative Approach to Infant Research: Promoting Reproducibility, Best Practices, and Theory-Building. Infancy, 22, 421-435. https://doi.org/10.1111/infa.12182

Henrich, J., Heine, S. J., \& Norenzayan, A. (2010). The weirdest people in the world. Behavioral and Brain Sciences, 33, 61-135. https://doi.org/10.1017/S0140525X0999152X

House, B. R., Silk, J. B., Henrich, J., Barrett, H. C., Scelza, B. A., Boyette, A. H., . . Laurence, S. (2013). Ontogeny of prosocial behavior across diverse societies. Proceedings of the National Academy of $\quad$ Sciences, 110(36), 14586-14591. https://doi.org/10.1073/pnas.1221217110 
Hood, B. M. (1995). Gravity rules for 2-to 4-year olds?. Cognitive Development, 10(4), 577-598. https://doi.org/10.1016/0885-2014(95)90027-6

Klein R. A., Ratliff K. A., Vianello M., Adams R. B.Jr., Bahník Š., Bernstein M. J., . . \& \& Nosek B. A. (2014). Investigating variation in replicability: A "many labs" replication project. Social Psychology, 45, 142-152. http://dx.doi.org/10.1027/1864-9335/a000178

Open Science Collaboration (2015). Estimating the reproducibility of psychological science. Science, 349(6251). https://doi.org/10.1126/science.aac4716

Safra, L., Tecu, T., Lambert, S., Sheskin, M., Baumard, N., \& Chevallier, C. (2016). Neighborhood deprivation negatively impacts children's prosocial behavior. Frontiers in Psychology, 7, 1760. https://doi.org/10.3389/fpsyg.2016.01760

Scott, K., Chu, J., \& Schulz, L. (2017). Lookit (Part 2): Assessing the Viability of Online Developmental Research, Results From Three Case Studies. Open Mind. https://doi.org/10.1162/OPMI_a_00001

Scott, K. M., \& Schulz, L. E. (2017). Lookit: A new online platform for developmental research. Open Mind. https://doi.org/10.1162/OPMI_a_00002

Sheskin, M., Nadal, A., Croom, A., Mayer, T., Nissel, J., \& Bloom, P. (2016). Some equalities are more equal than others: quality equality emerges later than numerical equality. Child Development, 87(5), 1520-1528. https://doi.org/10.1111/cdev.12544

Simmons, J. P., Nelson, L. D., \& Simonsohn, U. (2011). False-positive psychology: Undisclosed flexibility in data collection and analysis allows presenting anything as significant. Psychological Science, 22(11), 1359-66. https://doi.org/10.1177/0956797611417632 
Wagner, C. S., Whetsell, T. A., \& Leydesdorff, L. (2017). Growth of international collaboration in science: revisiting six specialties. Scientometrics, 110(3), 1633-1652. https://doi.org/10.1007/s11192-016-2230-9

Warneken, F., \& Tomasello, M. (2013). Parental presence and encouragement do not influence helping in young children. Infancy, 18(3), 345-368. https://doi.org/10.1111/j.15327078.2012.00120.x 


\section{Contributions}

Both authors contributed to the study concept and study design. MS collected the data, performed the analyses, and drafted the manuscript. FK provided critical revisions. Both authors approved the final version of the manuscript for submission.

\section{Funding Information}

Funding was provided by an NSF award (DRL-1561143) to FK and Yale Cognitive Science Research Funding provided to MS.

\section{Competing Interests}

The authors declare no competing interests.

\section{Data Accessibility Statement}

All the stimuli, presentation materials, and anonymized raw data can be accessed at osf.io/xdnuw 